\title{
1000 Scaffolding Technique: A New Technical Double-catheter Technique for Coil Embolization of Wide-necked Aneurysms
}

Jun Morioka, Kenichi Murao, Kousuke Miyake, and Hiroshi Miwa

Objective: We herein report a new technique using double microcatheters to treat a wide-necked aneurysm in which the neck is incorporated with the parent artery.

Case Presentations: Case 1: The patient was a 71-year-old woman with a large, wide-necked unruptured aneurysm of the basilar bifurcation area with the right posterior cerebral artery (PCA) incorporated in the aneurysm sac. We previously placed a scaffolding coil around the right PCA orifice via a microcatheter placed near the right PCA to avoid a framing coil via another microcatheter involving the right PCA orifice. After confirming that the framing coil did not obstruct the right PCA flow, the "scaffolding" coil was repositioned in the framing coil. Complete occlusion of the aneurysm was achieved with the stable frame of these two coils. Case 2: The patient was a 68-year-old woman who presented with subarachnoid hemorrhaging due to a ruptured aneurysm with a wide neck of the basilar bifurcation. Coiling with a conventional double-catheter technique failed to form suitable framing because the tips of both microcatheters faced the same direction (posterior) even after changing the shapes of the tips. After leading the tip of one microcatheter to face the anterior direction by inserting part of the first coil via the microcatheter, we placed the second coil via another microcatheter with its tip facing posteriorly. The second coil then came to functions as the scaffolding, holding the tip of the first catheter anteriorly. A stable frame was made when the remaining part of the first coil was deployed.

Conclusion: We termed this method the "scaffolding technique." This technique is an effective and safe option for treating wide-necked aneurysms.

Keywords $\$$ wide-necked aneurysm, double-catheter technique, coil embolization

\section{Introduction}

Embolizing an aneurysm with unfavorable configurations such as a wide neck or an important branch from the fundus remains technically challenging, despite advances in devices. Attempts to occlude such an aneurysm pose a risk

Department of Neuroendovascular Treatment, Shiroyama Hospital, Habikino, Osaka, Japan

Received: May 16, 2018; Accepted: November 2, 2018

Corresponding author: Jun Morioka. Department of Neuroendovascular Treatment, Shiroyama Hospital, 2-8-1 Habikino, Habikino, Osaka 583-0872, Japan

Email: morioka-nsu@umin.ac.jp

*The paper on the points of this argument was presented at the 32nd Annual Meeting of the Japanese Society for Neuroendovascular Therapy and the 40th Annual Meeting of the European Society of Neuroradiology.

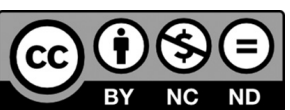

This work is licensed under a Creative Commons Attribution-NonCommercialNoDerivatives International License.

(C)2019 The Japanese Society for Neuroendovascular Therapy of coil herniation on the parent vessel. Stent-assisted coiling serves as a permanent buttress for the coil mass, but requires the long-term use of antiplatelet agents to prevent delayed in-stent stenosis. ${ }^{1-3)}$ Balloon-assisted coiling has the advantage of not requiring the long-term use of antiplatelet agents, but it serves as only a temporary buttress and may carry a higher risk of aneurysm perforation if the microcatheter impinges against the wall of the aneurysm during full inflation. $\left.{ }^{4}\right)$ We therefore use two microcatheters for coil embolization of some cerebral aneurysms with complicated configurations to make a stable coil frame.

We herein report a 71-year-old patient with an unruptured aneurysm at the top of the basilar artery and a 68-yearold patient with a ruptured aneurysm at the top of the basilar artery, who were successfully embolized with a modified microcatheter technique, which we have termed the "scaffolding technique." The aim of this report was to describe another possible treatment of a wide-necked aneurysm that may be suitable as an alternative to the balloon- or stentassisted method when conventional coiling fails. 

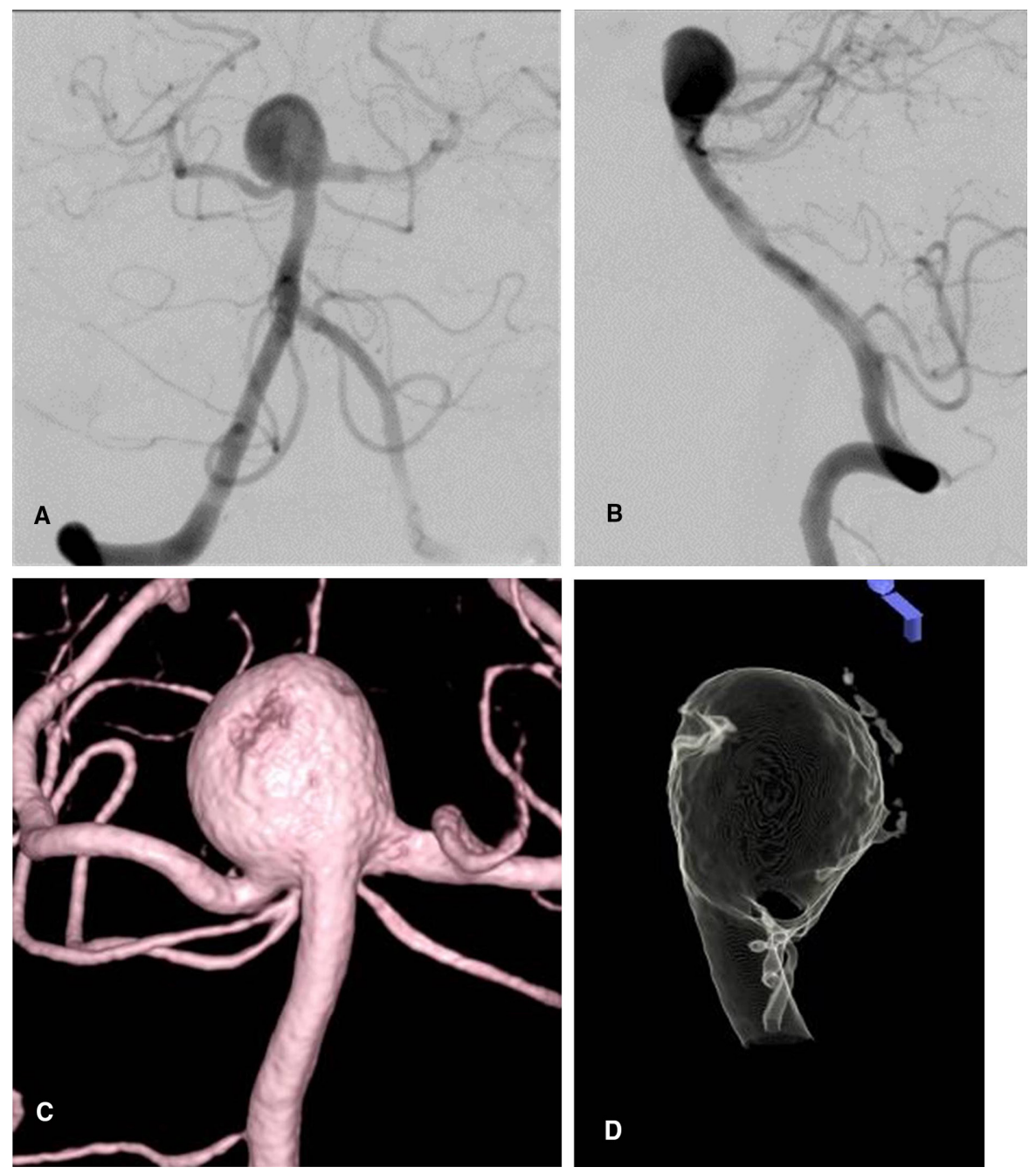

Fig. 1. Case 1: vertebral angiograms demonstrating the basilar tip aneurysm. (A) Anteroposterior view showing a large wide-necked aneurysm. The orifice of the right PCA is incorporated in the aneurysm sac. (B) Lateral view. The same projection as (D). (C) 3D image of the same aneurysm. Anteroposterior view. (D) Lateral projection with the bilateral PCA orifices superimposed in the translucent view. PCA: posterior cerebral artery

\section{Case Presentations}

\section{Case 1}

The patient was a 71-year-old woman. She consulted a local clinic with a headache. CT and MRI revealed an unruptured aneurysm of the basilar bifurcation area. She was referred to our hospital to undergo a detailed examination and treatment for the aneurysm. Cerebral angiography showed the basilar tip aneurysm whose size was $11.5 \times$ $10.5 \times 10.5 \mathrm{~mm}$, with a neck measuring $8.8 \mathrm{~mm}$. The orifice of the right posterior cerebral artery (PCA) was incorporated in the aneurysm sac (Fig. 1). Endovascular coil embolization was scheduled.

The oral administration of aspirin at $100 \mathrm{mg}$ /day and clopidogrel at $75 \mathrm{mg}$ /day was started 4 days before embolization. At first, we intended to use both a doublecatheter technique and a balloon-remodeling technique. Under general anesthesia, a 6 Fr sheath was placed in the right femoral artery, and a 5 Fr sheath was placed in the left femoral artery. Heparin at 3000 units in bolus and 1000 units per hour was systemically administered, with an activated clotting time of 230. A 6 Fr guiding catheter (Roadmaster; Goodman, Aichi, Japan) was guided into the right vertebral artery, and a 5 Fr guiding catheter was guided into the left vertebral artery. Via the 6 Fr guiding catheter, a microcatheter (Excelsior XT-17 Standard Straight; Stryker, Kalamazoo, MI, USA) steam shaped into a double angle and another straight microcatheter (Excelsior SL-10; Stryker) without steam shaping were guided into the aneurysm. We tried to navigate a balloon 

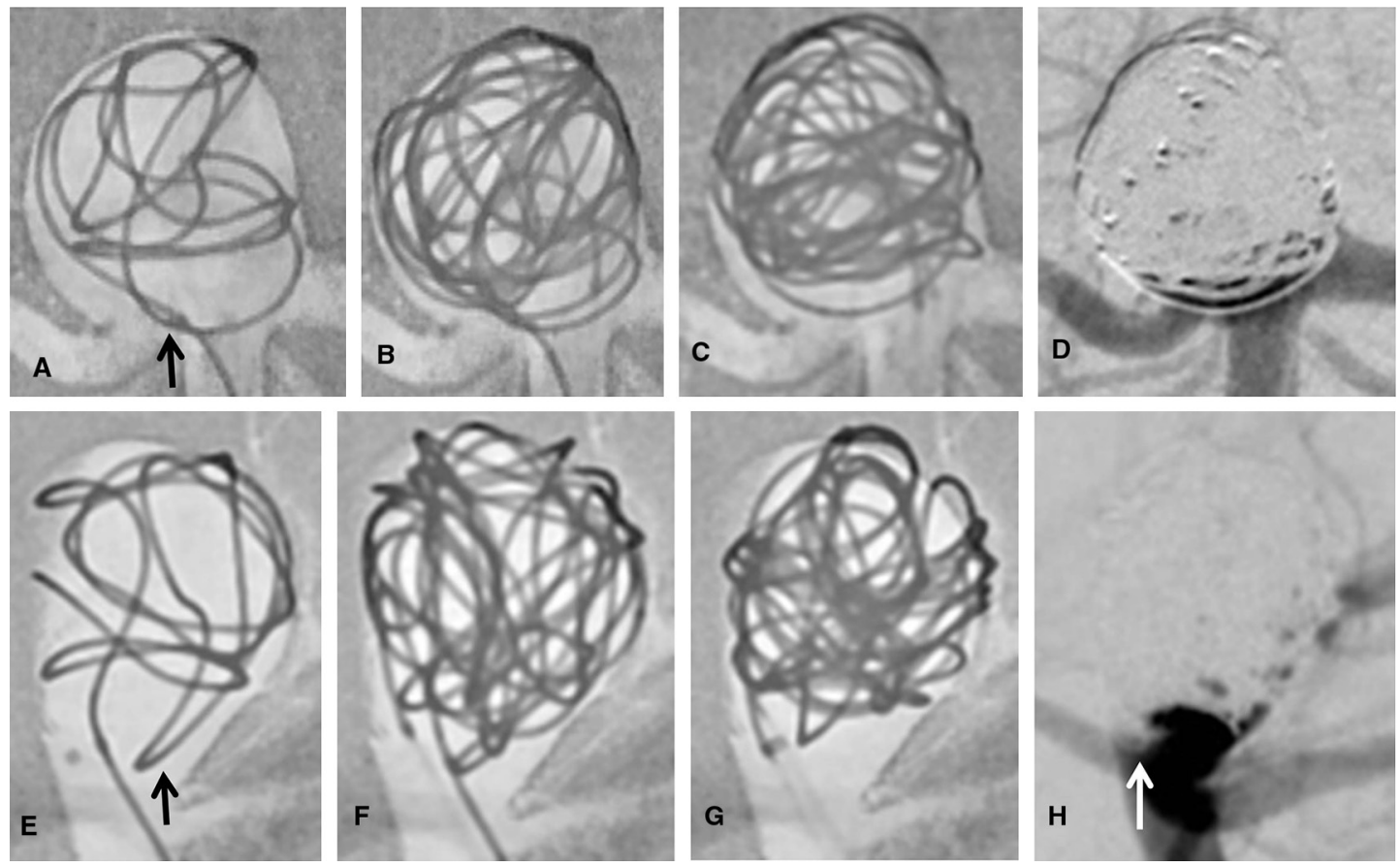

Fig. 2. Case 1. (A-D) Anteroposterior view. (E-H) Lateral view. (A and $\mathbf{E}) \mathrm{A}$ "scaffolding" coil is partially deployed. The arrows in $\mathbf{A}$ and $\mathbf{D}$ show the coil loop herniated on the right PCA. (B and $\mathbf{F}$ ) The framing coil via another catheter is completely deployed. (C and $\mathbf{G}$ ) After confirming no impingement of the framing coil loops on the parent artery, the herniated coil is repositioned into the framing coil. ( $\mathbf{D}$ and $\mathbf{H})$ The final angiograms show almost complete occlusion. The arrow in $\mathbf{H}$ shows the coil mass in the inflow zone. PCA: posterior cerebral artery

catheter via the $5 \mathrm{Fr}$ guiding catheter into the right PCA, but the delivery was prevented by the acute angle between the basilar artery and the right PCA. Therefore, we switched our approach to coiling using only the doublecatheter technique. The tip of the XT-17 microcatheter was placed near the right PCA orifice. The tip of the SL-10 microcatheter was placed within the aneurysm lumen. To avoid the framing coil (Microplex-18 $12 \mathrm{~mm}$; Terumo Corporation, Tokyo, Japan) from the SL-10 catheter becoming involved with the right PCA orifice, another coil (Microplex-18 $10 \mathrm{~mm}$; Terumo) from the XT-17 catheter was partially inserted around the right PCA orifice in advance. One loop of the coil (the scaffolding coil) was intentionally herniated on the right PCA orifice (Fig. 2A and 2E). Then, the framing coil was completely deployed (Fig. 2B and $\mathbf{2}$ F). After confirming that the framing coil did not obstruct the right PCA flow, the scaffolding coil was repositioned in the framing coil (Fig. 2C and 2H). Some filling coils were introduced into the framing coil. At the last stage, we embolized the inflow zone near the PCA orifices, by referencing the lateral projection with the bilateral PCA orifices superimposed onto the translucent view (Figs. 1D and 2H). Almost complete occlusion was achieved (Figs. 2D, 2H and 3).

\section{Case 2}

The patient was a 68 -year-old woman. She consulted a local clinic with a sudden headache. The headache developed suddenly 7 days before the consultation but improved once. Examinations for the cause of the headache revealed subarachnoid hemorrhaging and a basilar tip aneurysm. She was transferred to our hospital by ambulance. The Japan Coma Scale score was 0, and the Glasgow Coma Scale was 15. The Hunt and Hess grade was evaluated as 2, the World Federation of Neurological Surgeons grade as 1, and Fisher group as 3. On the day of admission, cerebral angiography revealed the basilar tip aneurysm of $7.5 \times$ $4.9 \times 4.2 \mathrm{~mm}$ in size with a neck measuring $4.7 \mathrm{~mm}$ (Fig. 4A and 4B). The depth of the aneurysm was narrow, and it was long in the longitudinal direction. Endovascular coil embolization was scheduled for the next day.

Under general anesthesia, a 6 Fr sheath was placed in the right femoral artery. Heparin at 3000 units in bolus and 1000 units per hour was systemically administered, with an activated clotting time of 250. A 6 Fr guiding catheter (Roadmaster) was guided into the right vertebral artery. An Excelsior SL-10 (preshaped $45^{\circ}$ ) and another straight microcatheter (Headway-17; Terumo) were guided into the aneurysm via the same guiding catheter. The tips of both 

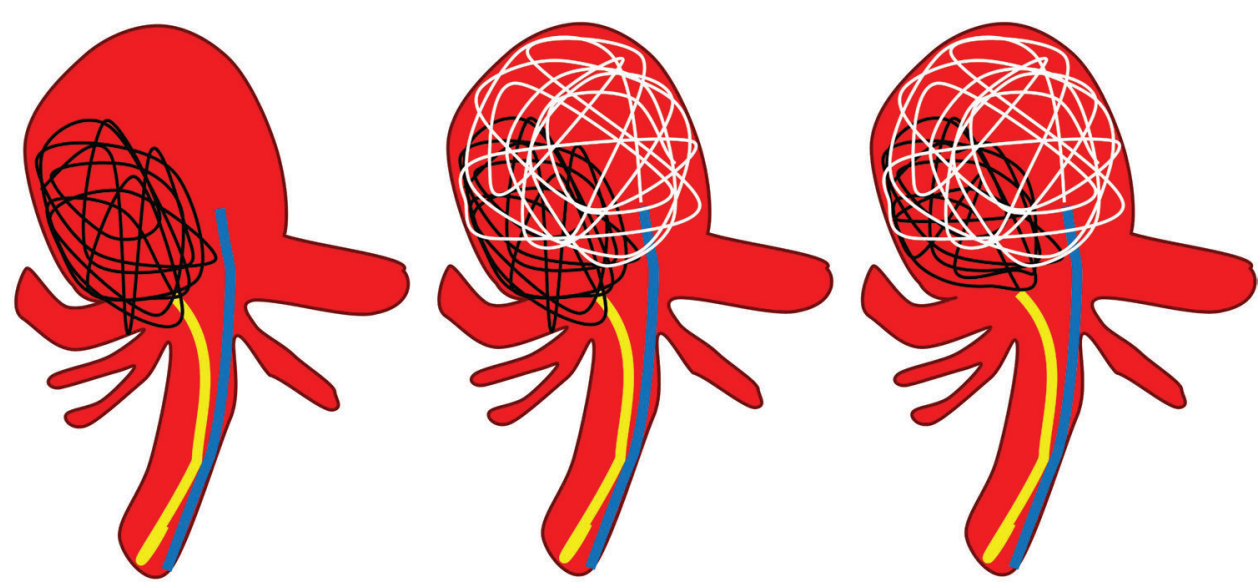

Fig. 3. A schematic illustration of the scaffolding technique of case 1. The tip of a microcatheter (MC1) is placed near the right PCA orifice. The tip of another microcatheter (MC2) is placed within the aneurysm lumen. To avoid the framing coil via MC2 becoming involved with the right PCA orifice, another scaffolding coil from MC1 is placed around the right PCA orifice. After confirming no impingement of the framing coil loops on the parent artery, the scaffolding coil is repositioned in the framing coil. PCA: posterior cerebral artery
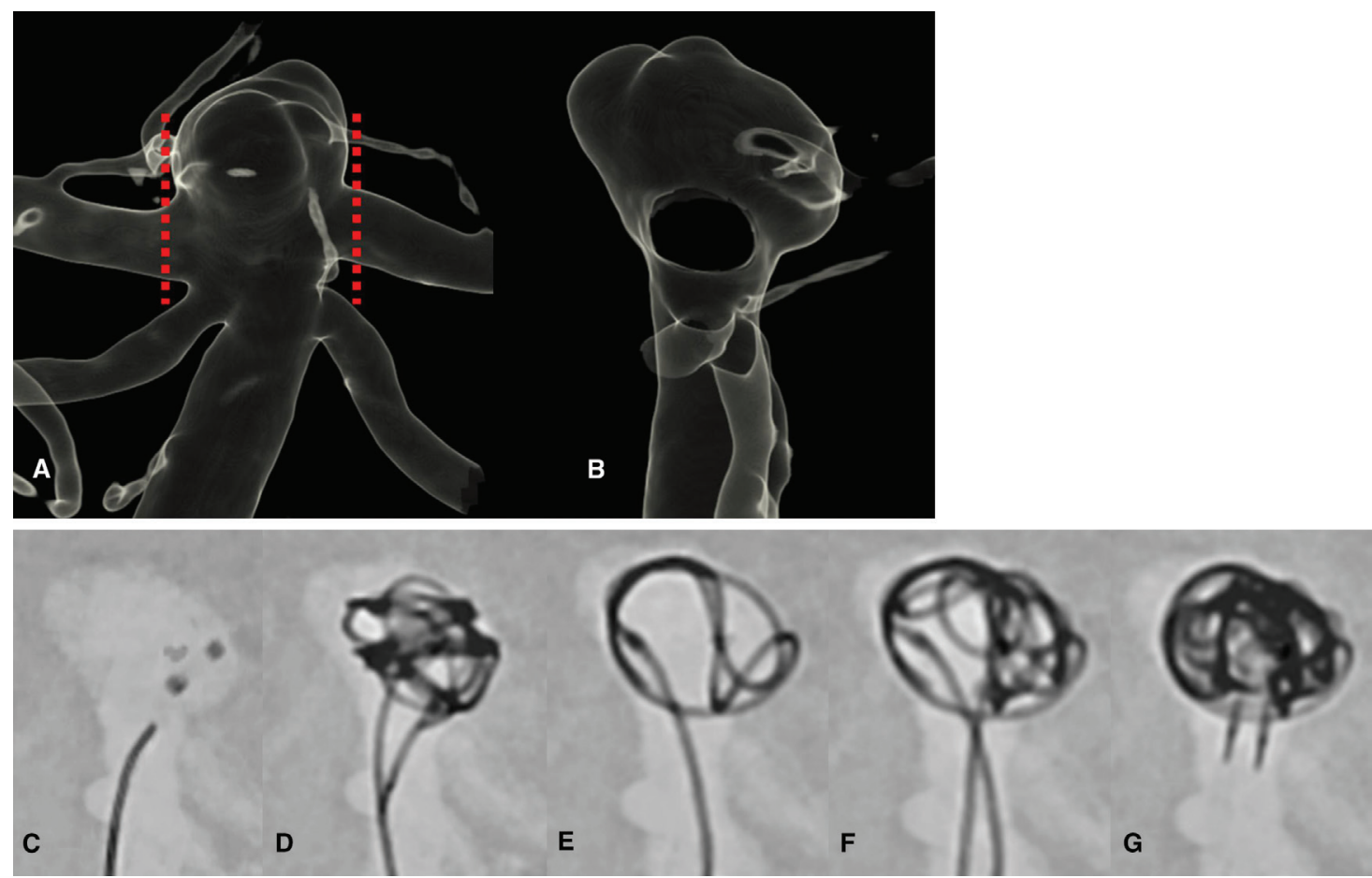

Fig. 4. Case 2: vertebral angiograms demonstrating the basilar tip aneurysm. (A) Anteroposterior view showing a large, widenecked aneurysm. The dotted red lines show the bilateral PCA orifices. (B) Lateral projection with the bilateral PCA orifices cut with the dotted red lines in (A) superimposed in the translucent view. (C-G) Lateral view with the same projection as (B). (C) The tips of both microcatheters faced the posterior direction. (D) Two coils (Orbit Galaxy fill $6 \mathrm{~mm} \times 10 \mathrm{~cm}$ and $5 \mathrm{~mm} \times 10 \mathrm{~cm}$; Johnson \& Johnson Codman, Miami, FL, USA) via each microcatheter were initially tried, but both coils occupied the same posterior part of the aneurysm and compromised the parent vessel. (E) The tip of a microcatheter faced anteriorly when part of the first coil (Orbit Galaxy fill $6 \mathrm{~mm} \times 10 \mathrm{~cm}$ ) was inserted via the microcatheter. $(\mathbf{F})$ The second coil (Orbit Galaxy fill $5 \mathrm{~mm} \times 10 \mathrm{~cm}$ ) inserted via another microcatheter with its tip facing posteriorly. (G) A remaining part of the first coil was deployed. A stable frame was made. PCA: posterior cerebral artery 


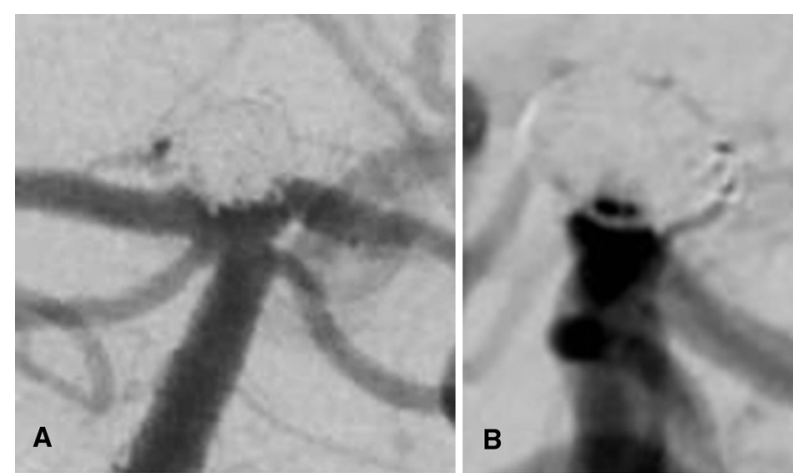

Fig. 5. Case 2: the final angiograms showing almost complete occlusion. (A) Anteroposterior view and (B) lateral view.

microcatheters faced the posterior direction even after we changed the tip shape because the direction of the tip was fixed by the angioarchitecture of the proximal artery (Fig. 4C). Two coils (Orbit Galaxy fill $6 \mathrm{~mm} \times 10 \mathrm{~cm}$ and $5 \mathrm{~mm} \times 10 \mathrm{~cm}$; Johnson \& Johnson Codman, Miami, FL, USA) via each microcatheter were initially tried but removed because both coils occupied the same posterior part of the aneurysm and compromised the parent vessel (Fig. 4D). The tip of the Excelsior SL-10 microcatheter faced anteriorly when part of the first coil (Orbit Galaxy fill $6 \mathrm{~mm} \times 10 \mathrm{~cm}$ ) was inserted via the microcatheter (Fig. 4E). While leaving the first coil alone, we inserted the second coil (Orbit Galaxy fill $5 \mathrm{~mm} \times 10 \mathrm{~cm}$ ) via the Headway-17 with its tip facing posteriorly (Fig. 4F). The second coil was completely deployed and occupied the posterior part of the aneurysm. The tip of the Excelsior SL-10 was held anteriorly even while a remaining part of the first coil was deployed. A stable frame was made (Fig. 4G) and almost complete occlusion was achieved (Fig. 5).

\section{Discussion}

In this report, we demonstrated a brand-new double-catheter technique, named the "scaffolding technique." The key concept of this scaffolding technique is making a coil frame sustained by another coil. The scaffolding coil is repositioned into the framing coil when it compromises the parent vessel.

The double-catheter technique was first described in 1998 by Baxter et al.,5) and a few authors ${ }^{6-9)}$ have reported on the effectiveness of the technique. The concept of their method was different from that of our scaffolding technique. Their concept involved making a stable coil frame with two coils through two microcatheters. When there was a risk of coil migration because a coil smaller than the aneurysmal sac had to be initially placed without compromising the parent vessel, they inserted the second coil through the second microcatheter to form a more stable coil mesh. However, even their standard double-catheter technique may fail to make a desirable coil frame for an aneurysm with a too-wide neck due to coil protrusion into the parent vessel. Furthermore, when the branch artery is small and departs from parent vessel at an acute angle, neck-bridging stent or balloon is dangerous, causing hazards such as aneurysmal rupture, vasospasm, arterial dissection, and flow arrest. In such cases, our scaffolding technique may prove successful. A coil may be intentionally left protruding into the parent vessel as a scaffold like in our case 1, with another coil used as a frame. A scaffolding coil functioned as a temporary neck-bridging stent or balloon in our case 1 . In contrast, in our case 2, a scaffolding coil stabilized a microcatheter through which another coil was inserted. Unsatisfactory catheter positioning was able to be overcome using the scaffolding coil. This is a point unlike simple double-catheter technique. In both cases, a coil served as a scaffold for another coil to be deployed.

Because coil stability is achieved with the first two coils, it is important to consider coil selection (size, length, and shape). In our cases, the maximum coil size was selected based on the average of the minimum and maximum length of the aneurysm. The longer a coil is, the more stable the resultant frame. However, it is usually difficult to use a long coil in especially difficult situations in which the scaffolding technique applies. Regarding the coil shape, three-dimensional coils were preferred in our cases. Three-dimensional coils are useful for making a coiling frame for wide-necked aneurysms, as it is easy to bridge the aneurysmal neck with coil loops. ${ }^{10)}$ Three-dimensional coils can be classified based on their convergence: inward from the outside or expanding outward and which three-dimensional coils are most suitable for the scaffolding technique depend on the situation. For example, three-dimensional coils that expand outward are suitable for multi-lobulated or spindly aneurysms ${ }^{11)}$ but trend to compromise the parent vessels in cases of wide-necked aneurysms. If an aneurysmal neck is protected with a scaffolding coil, as in our case 1, a coil expanding outward can be used as the second coil. Of note, it is difficult to use a coil expanding outward as a scaffold in cases where it needs to remain inside an aneurysmal sac.

The technique that we have proposed has some limitations. It is not usually easy to make a frame without impingement of the frame coil loops on the parent artery 
even when there is a scaffolding coil. If it takes a long time to make a desirable frame leaving a scaffolding coil herniated on the parent vessel, the risk of thromboembolic complication may be increased. A preprocedural preparation with dual antiplatelets may lower the risk of thromboembolism. The restrictive use of a scaffolding coil herniated on the parent vessel should be recommended in patients with a rupture aneurysm. Furthermore, there is also a risk of forming a tangle with these two coils, sometimes followed by unraveling. Several attempts at coil advancement or retrieval may cause the two coils to become interlocking. To avoid such a situation, the second coil should be deployed after the complete deployment of the first. When it is never made, as in our case 1 and case 2, coil advancement and retrieval should be little as possible. However, as long as the initial two coils are not detached and the both coils are not unraveled, they can be controlled and usually retrieved if the two microcatheters are in the same guiding catheter in parallel. ${ }^{7)}$ It is important to notice quickly when one coil is unraveled before the both fail. But, neither tangles nor unraveling were noted in the actual treatment in either of our cases. Although we do not recommend this technique as a first-line method for coil embolization, it may serve as a reliable alternative for coiling of a wide-necked aneurysm in situations in which standard methods cannot be applied.

\section{Conclusion}

This scaffolding technique may be an effective and safe therapeutic option for wide-necked aneurysms instead of permanent placement of a stent in the parent artery.

We presented the paper on the points of this argument at the 32nd Annual Meeting of the Japanese Society for Neuroendovascular Therapy (November 24-26, 2016, Japan) and the 40th Annual Meeting of the European Society of Neuroradiology (September 13-17, 2017, Sweden). ${ }^{12)}$

\section{Disclosure Statement}

The authors report no conflicts of interest regarding this article.

\section{References}

1) Lee YJ, Kim DJ, Suh SH, et al: Stent-assisted coil embolization of intracranial wide-necked aneurysms. Neuroradiology 2005; 47: 680-689.

2) Kim DJ, Suh SH, Kim BM, et al: Hemorrhagic complications related to the stent-remodeled coil embolization of intracranial aneurysms. Neurosurgery 2010; 67: 73-78.

3) Piotin M, Blanc R, Spelle L, et al: Stent-assisted coiling of intracranial aneurysms: clinical and angiographic results in 216 consecutive aneurysms. Stroke 2010; 41: 110-115.

4) Malek AM, Halbach VV, Phatouros CC, et al: Balloon-assist technique for endovascular coil embolization of geometrically difficult intracranial aneurysms. Neurosurgery 2000; 46: 1397-1406.

5) Baxter BW, Rosso D, Lownie SP: Double microcatheter technique for detachable coil treatment of large, wide-necked intracranial aneurysms. AJNR Am J Neuroradiol 1998; 19: $1176-1178$.

6) Murao K, Taki W, Kawaguchi K, et al: Interventional radiologic techniques for basilar bifurcation aneurysms. Techniques in Neurosurgery 2000; 6: 234-237.

7) Kwon OK, Kim SH, Kwon BJ, et al: Endovascular treatment of wide-necked aneurysms by using two microcatheters: techniques and outcomes in 25 patients. AJNR Am J Neuroradiol 2005; 26: 894-900.

8) Kim DJ, Kim BM, Park KY, et al: Coil embolization of overwide and undertall small intracranial aneurysms with double microcatheter technique. Acta Neurochir (Wien) 2014; 156: 839-846.

9) Xu X, Zheng Y, Wang D, et al: Improved endovascular coiling of wide-neck intracranial aneurysms in elderly patients by double-microcatheter technique. Cell Biochem Biophys 2015; 71: 1281-1286.

10) Vallée JN, Pierot L, Bonafé A, et al: Endovascular treatment of intracranial wide-necked aneurysms using three-dimensional coils: predictors of immediate anatomic and clinical results. AJNR Am J Neuroradiol 2004; 25: 298-306.

11) Sugiu $K$, Tokunaga $K$, Nishida $A$, et al: [Clinical experiences of new detachable coils in the treatment of cerebral ameurysm Micruspere \& Microplex.] JNET 2007; 1: 45-53. (in Japanese)

12) Murao K, Morioka J, Miwa H: Scaffolding technique a new double catheter technique in the coil embolization of wide necked aneurysms. ESNR 2017. Neuroradiology 2017; 59(Suppl 1): 98. 